\title{
Une modélisation d'accompagnement pour la gestion combinée des systèmes d'élevage et des milieux boisés sur le Causse du Larzac
}

\author{
Michel Étienne ${ }^{1}$ \\ Laurent Dobremez ${ }^{2}$ \\ Gérard Guerin ${ }^{3}$ \\ Hélène Rapey ${ }^{4}$ \\ Christophe Simon ${ }^{5}$ \\ ${ }^{1}$ Inra \\ Unité d'écodéveloppement \\ Site Agroparc \\ 84914 Avignon \\ France \\ <etienne@avignon.inra.fr> \\ ${ }^{2}$ Cemagref \\ Unité Développement des territoires \\ montagnards \\ Saint-Martin d'Hères \\ France \\ <laurent.dobremez@cemagref.fr> \\ ${ }^{3}$ Institut de l'élevage \\ Montpellier \\ France \\ <gerard.guerin@inst-elevage.asso.fr> \\ ${ }^{4}$ Cemagref \\ UMR Métafort \\ Aubière \\ France \\ <helene.rapey@cemagref.fr> \\ ${ }^{5}$ Inra \\ Unité d'écodéveloppement \\ Avignon \\ France \\ <christophe.simon@gmx.com>
}

\begin{abstract}
Résumé
Après une présentation des conditions qui ont mené à la constitution de la Société cvile des terres du Larzac (SCTL), la démarche de modélisation d'accompagnement utilisée avec ce collectif d'éleveurs est présentée. Le modèle coconstruit par un groupe de techniciens et de chercheurs est décrit, ainsi que ses modalités d'utilisation avec les acteurs locaux, pour confronter des scénarios d'aménagement, envisagés par la SCTL dans une optique de gestion du massif forestier, à ceux qui ont été élaborés par les éleveurs pour améliorer le fonctionnement de leurs exploitations.
\end{abstract}

Mots clés : aménagement forestier ; France ; modélisation environnementale ; système agrosylvopastoral.

Thèmes : méthodes et outils ; productions animales ; ressources naturelles et environnement ; territoire, foncier, politique agricole et alimentaire.

\section{Abstract}

A model to accompany the combined management of livestock farming systems and woodlands on the Causse du Larzac (Aveyron, France)

Once the conditions that led to the creation of the Société Civile des Terres du Larzac (SCTL) have been introduced, the companion modelling approach used with this group of farmers is presented. The agent-based model co-constructed with a group of researchers and technicians is then described, as well as the way it was used to confront scenarios developed by the SCTL to envision management options of the forest massif, with scenarios developed by the farmers to improve the current functioning of their farms.

Key words: agrosilvopastoral systems; environmental modelling; forest management; France.

Subjects: animal productions; forests; natural resources and environment; tools and methods; territory, land use, agricultural and food policy.
C oncilier des modes d'utilisation de l'espace raisonnés au niveau d'exploitations agricoles et une gestion durable de l'espace forestier à l'échelle d'un territoire est un enjeu et une difficulté récurrente dans beaucoup de territoires à faible productivité. Les difficultés portent notamment sur les interactions à prendre en compte: entre différentes échelles spatiales et temporelles, entre décisions technico-économiques et processus écolo- giques, entre logiques individuelles et collectives... Pour représenter les interactions entre dynamiques écologiques et dynamiques sociales sur un territoire, de nombreux travaux de modélisation se sont fondés sur les systèmes multi-agents (SMA) (Franc et Sanders, 2000). Le travail présenté dans cet article s'inscrit dans une démarche de modélisation d'accompagnement (Collectif Commod, 2006) pour une gestion sylvopastorale d'un massif forestier. 
Contexte de la situation étudiée

Après dix années de " lutte " des éleveurs, le projet d'extension du camp militaire du Larzac est abandonné en mai 1981. Au cours de cette période, des groupements fonciers agricoles (GFA) ont été créés pour acquérir des terres (1 200 hectares pour 2700 apporteurs de parts) et contrecarrer les achats et expropriations par l'État (6 300 hectares). En 1985, la Société civile des terres du Larzac (SCTL) est créée et se voit confier par l'État, par bail emphytéotique, la gestion de ces 6300 hectares (De Crisenoy et Boscheron, 1986). La SCTL 85 associés et un conseil de gérance composé de 7 éleveurs, 2 usagers non agricoles et 2 membres de commissions communales -, qui gère ce foncier en commun avec les GFA Larzac, a conclu des baux de carrière avec ses "fermiers " composés d'anciens éleveurs et de néoruraux. L'un des enjeux locaux, exprimé par les gérants de la SCTL dès 1997, est la fermeture progressive de l'espace pastoral par les accrus de pin sylvestre et par la densification du couvert dans les formations boisées de chêne pubescent anciennement pâturées et exploitées pour le bois de chauffage. En effet, tandis que le pâturage et l'entretien des terres achetées par l'État étaient interrompus, les systèmes d'élevage ovins-lait pour la filière Roquefort se sont fortement transformés, en se repliant sur des surfaces fourragères (prairies temporaires et céréales) conduites de façon relativement intensive.

En 1998, les gérants de la SCTL font appel à un expert forestier pour réaliser un plan simple de gestion (PSG), obligatoire pour les propriétaires forestiers privés possédant plus de 25 hectares de forêt. Ce plan ne répondant pas à leurs attentes, ils envisagent les possibilités de gestion de leurs bois par le sylvopastoralisme et se rapprochent alors de l'Institut de l'élevage et de l'Institut pour le développement forestier. La démarche mise en ouvre par l'Institut national de recherche agronomique (Inra) sur le Causse Méjan voisin (Étienne et al., 2003) leur est présentée et ils se portent volontaires pour l'appliquer à leur situation.

La question, posée par la SCTL, d'une meilleure valorisation des bois est retravaillée par un collectif regroupant des spécialistes d'instituts techniques et des chercheurs travaillant sur les systèmes d'élevage, le sylvopastoralisme et la modélisation d'accompagnement. La question est alors recentrée sur la façon de concilier les gestions individuelles des fermiers sur leurs exploitations agricoles et une gestion collective des bois organisée par la SCTL à l'échelle du massif forestier. Il fallait, pour y répondre, regrouper les connaissances disponibles et aider les acteurs locaux à confronter leurs connaissances avec les représentations produites par le modèle, puis à " jouer avec le modèle " pour imaginer des options de mise en valeur de leur territoire.

\section{Caractérisation du territoire et des systèmes d'élevage}

Les 8800 hectares modélisés se situent au nord-est du Causse du Larzac, plateau karstique (700 à 1000 mètres d'altitude) au sud du Massif central. Ils sont utilisés par 35 fermiers et se répartissent en 4250 hectares de parcours, 3200 hectares de forêts et 1350 hectares de cultures. Les zones boisées se composent de 900 hectares de futaies de pin sylvestre dont $75 \%$ concentrés sur cinq exploitations agricoles -, le reste étant formé de parcours enrésinés et de taillis et parcours boisés en chêne pubescent.

L'élevage ovin laitier est l'activité agricole dominante (18 exploitations sur 35) mais ce territoire couvre une grande diversité de systèmes de production - ovinsviande, caprins-lait, bovins-viande, chevaux pour spectacles de cirque, volailles, plantes aromatiques pour la distillation et l'importance des combinaisons d'activités fait entrevoir l'acuité de la question de l'organisation du travail : dans plus de la moitié des exploitations, la production agricole, la commercialisation en circuits courts, l'artisanat ou des activités non agricoles hors de la ferme sont pratiqués par les membres de la cellule de base (Dedieu et al., 2006). Or, ces activités doivent être articulées avec la conduite des cultures et des troupeaux, très exigeante en travail, en particulier en système d'élevage ovin-lait. La taille des troupeaux varie de 90 à 600 brebis par exploitation et la surface de 160 à 570 hectares, dont 0 à 190 ha de cultures, 70 à 540 ha de prairies et parcours boisés, et 5 à 210 ha de forêts (taux de recouvrement arboré supérieur à 50 \%).

\section{Élaboration du modèle}

Notre modèle articule un modèle agroécologique et un modèle à base de règles de décision prêtées aux acteurs, et projette les résultats sur un automate cellulaire qui permet de rendre compte de la dynamique spatiale des couverts végétaux.

\section{Modèle conceptuel}

La première étape de la démarche a consisté à représenter le système socioécologique en identifiant les principales dynamiques écologiques en rapport avec la gestion de la végétation boisée, puis à inventorier les agents susceptibles d'avoir un impact : i) direct, par leurs pratiques sur les dynamiques écologiques; ou ii) indirect en orientant les choix de gestion d'autres agents (Étienne et al., 2003). Par rapport au modèle Méjan, les principales adaptations ont porté sur les logiques des acteurs modélisées (ici les fermiers et l'acteur collectif SCTL) et l'introduction des dynamiques écologiques du chêne pubescent et de la dimension organisation du travail dans les systèmes d'élevage.

La comparaison des photos aériennes en 1948 et en 1997 montre une importante fermeture des milieux pâturés et cultivés par colonisation d'espèces ligneuses (Simon, 2004). Ce processus assimilable à une succession secondaire a favorisé l'expansion du pin sylvestre et du chêne pubescent, dont les processus de colonisation et les interactions avec les pratiques agropastorales ont été décrits (Lepart et al., 1999; Étienne, 2001). La dynamique des couverts végétaux en fonction des modes de gestion a été retranscrite sur un diagramme de transition (figure 1).

Les acteurs susceptibles de jouer un rôle dans la gestion de la végétation boisée ont été identifiés à partir d'une pré-enquête auprès de la SCTL. Chaque agent est décrit par ses entités de gestion, un certain nombre d'attributs et des pratiques ou actions (figure 2).

\section{Autres étapes clés de l'élaboration du modèle}

Les mailles élémentaires spatio-temporelles ont été définies afin de rendre correctement compte de l'hétérogénéité des entités de gestion (pixel de 1 hectare) et des rythmes liés à l'organisation du travail (pas de temps d'une semaine). 


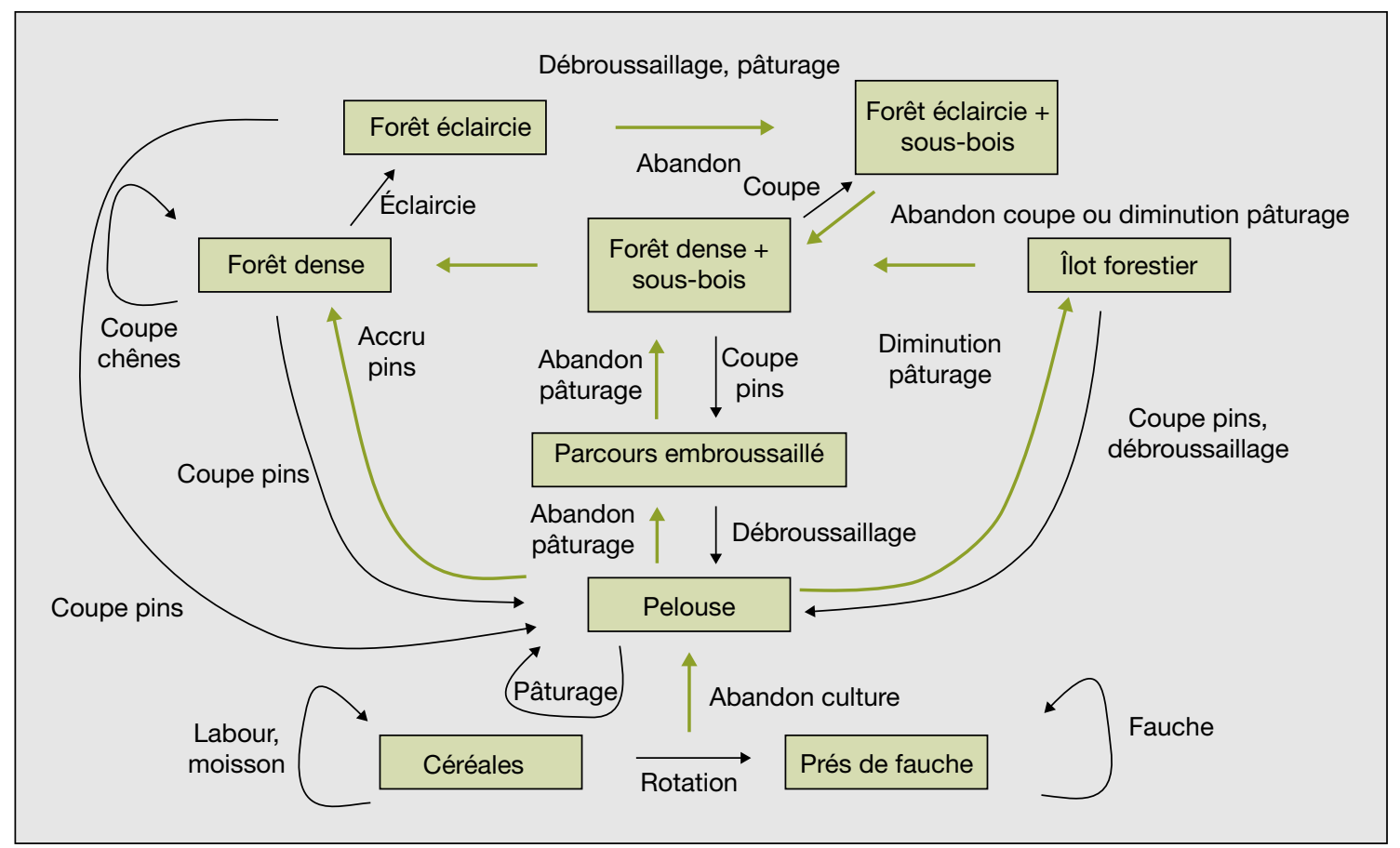

Figure 1. Diagramme de transition: dynamiques des couverts végétaux selon différentes modalités d'intervention.

Figure 1. Transition diagram: vegetation dynamics according to farmers' practices.

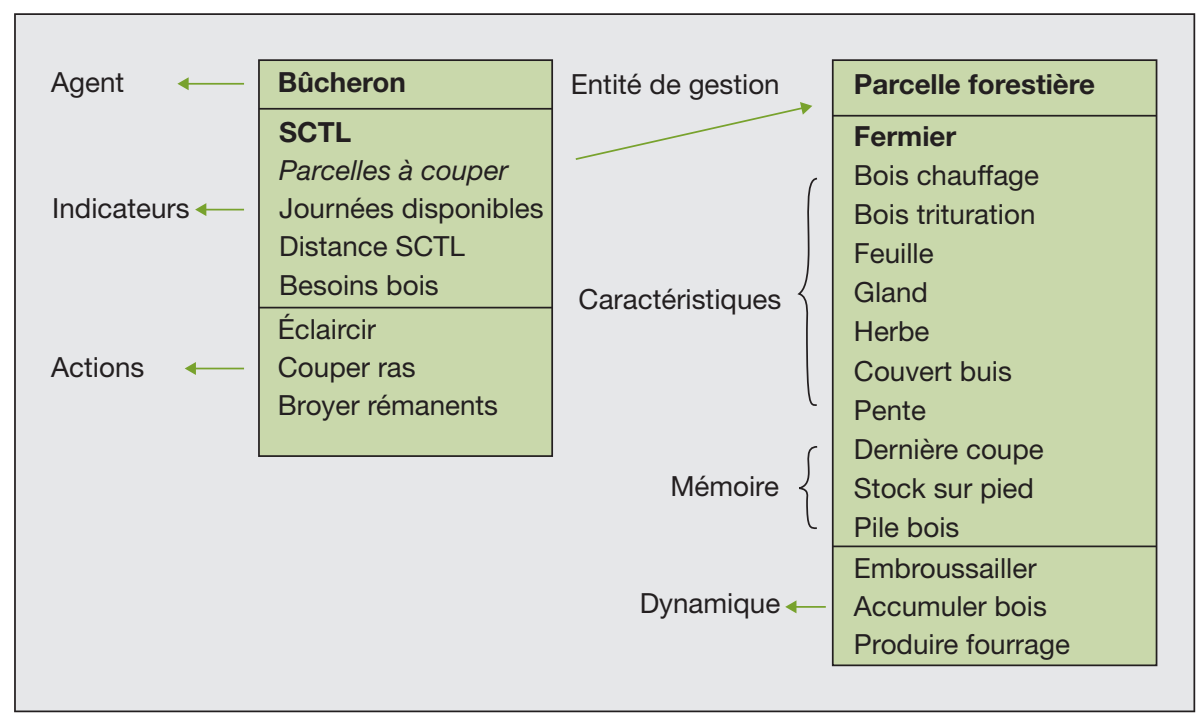

Figure 2. Attributs et actions de l'agent bûcheron et de ses parcelles de coupe.

Figure 2. Attributes and procedures of the logger and his forest plots. SCTL : Société civile des terres du Larzac.

\section{Modéliser les dynamiques végétales}

La description de la végétation boisée s'appuie sur les données du PSG (type de peuplement, espèce dominante). Ces données étant incomplètes, nous avons estimé lâge des peuplements à partir du taux de recouvrement boisé et en identifiant les essences dominantes, et le volume du peuplement à partir des données d'accroissement de l'Inventaire forestier national (région des Grands Causses). La caractérisation du sous-bois a été faite par estimation du recouvrement sur les photos aériennes de 1997 et des observations de terrain complémentaires : espèce arbustive dominante ; ressource pastorale.

La dynamique de fermeture du milieu a été établie en fonction des taux de recouvrement arboré, arbustif et herbacé mesurés sur une centaine de points sur la série de 5 missions aériennes disponibles depuis 1948 (Simon, 2004). Des matrices de transition et des modèles de croissance des peuplements forestiers ont alors été développés à partir des pratiques pastorales et sylvicoles locales (coupe rase ou sélective) et de dires d'experts.

À partir des références pastorales disponibles (Guérin et al., 2001), des courbes de croissance saisonnière de l'herbe ont été modélisées, ainsi que la disponibilité en glands dans les chênaies. Un simulateur climatique reproduit la variabilité des saisons observée au cours des 20 dernières années.

\section{Modéliser les pratiques} et décisions des éleveurs

Les données sur les fermiers combinent des caractéristiques individuelles (nature des activités, taille des troupeaux, entités de gestion, collectif de travail présent sur 
l'exploitation...) et des règles de décision pour l'utilisation de l'espace et l'organisation du travail en fonction des systèmes d'élevage.

Sur les 35 fermiers disposant de bois appartenant à la SCTL, 31 ont été enquêtés. L'enquête visait à comprendre la logique globale de fonctionnement de l'exploitation et à préciser les pratiques en liaison avec l'organisation spatiale de l'exploitation, l'organisation du travail et la disponibilité en surfaces boisées :

- définir et localiser les entités de gestion et leurs usages (cultures, surfaces fourragères, parcours, bois) ;

- caractériser la conduite des troupeaux : périodes de mise bas et de production laitière, choix d'allotement et effectifs par lots ;

- identifier les plannings de pâturage par lots et les parcs concernés ;

- préciser les fonctions attendues des surfaces boisées, les pratiques sylvicoles et les besoins en bois, ainsi que les changements que les pratiques sylvicoles pourraient entraîner sur l'organisation du pâturage ;

- élaborer un calendrier d'activités sur une campagne annuelle pour les membres de la cellule de base.

Les formations végétales, le territoire d'exploitation, les parcs, l'emplacement des bergeries et le mode de pâturage ont été spatialisés et saisis sur un système d'information géographique, puis exportés sur la plateforme CORMAS ${ }^{1}$ (Bousquet et al., 1998).

Des règles ont ensuite été définies pour l'affectation des lots d'animaux aux parcs. Pour la main-d'œuvre, des ordres de priorité ont été établis: une fois déduit le nombre de journées consacrées aux activités non liées à la conduite du troupeau, des règles ont été appliquées pour évaluer le temps à consacrer au troupeau et aux surfaces pastorales, tâches considérées comme prioritaires par rapport aux pratiques sylvicoles. Enfin, quand il reste au moins trois jours disponibles dans la semaine, des règles de sélection des parcelles de coupe de bois sont appliquées, selon le besoin en bois de chauffage et les ressources pastorales disponibles sur l'exploitation (Simon, 2004).

\footnotetext{
${ }^{1}$ Logiciel libre, accessible sur le site http://cormas.cirad.fr/.
}

\section{Scénarios testés}

\section{Simulations}

Chaque simulation porte sur une succession de 40 années climatiques reproduisant la fréquence passée d'années sèches, moyennes ou bonnes, mais dont l'ordre est généré de façon aléatoire. Au cours d'un cycle annuel, le modèle donne priorité à l'alimentation des troupeaux puis détermine si les agents vont exercer des pratiques sylvicoles selon l'état de la ressource boisée, les besoins et la force de travail disponible. Il simule alors la dynamique de la végétation en fonction des caractéristiques climatiques de l'année et des pratiques pastorales et sylvicoles mises en ouvre.

\section{Scénarios}

La discussion sur les simulations du modèle s'est tenue en trois temps. Le groupe de travail a d'abord organisé une présentation à la SCTL et à un groupe de fermiers de la simulation du prolongement de la gestion actuelle (scénario 1). Cette séance a permis de valider le modèle et de faire prendre conscience de la nécessité d'intervenir fortement sur l'espace forestier pour endiguer la progression de la forêt. Il a alors été proposé aux fermiers volontaires de mettre sur papier leurs projets d'exploitation forestière.

Les fermiers et la SCTL ont alors souhaité disposer de données forestières à l'échelle des exploitations pour préciser leurs projets. À la fin de l'exercice, 11 fermiers se sont opposés à l'exploitation de leur forêt, car ils la considèrent en équilibre avec leur activité d'élevage, et leurs besoins en bois de chauffage. Quatre fermiers ne ressentent pas le besoin d'aménager leur forêt, soit parce qu'ils ne sont pas en manque de fourrage, soit parce qu'ils considèrent que la fermeture du couvert forestier peut pallier les effets de trois années successives de sécheresse. Dix fermiers souhaitent aménager leur forêt, soit parce qu'ils sont confrontés à une forte dynamique du pin sylvestre et souhaitent en limiter les effets, soit parce qu'ils disposent de vieux peuplements de chêne blanc dont ils souhaitent mieux valoriser la production de bois et de fourrage. Enfin, six fermiers ont un besoin vital d'intervention dans leur forêt pour récupérer des zones de pâturage. Une deuxième séance de simulation a permis d'évaluer l'impact de la mise en œuvre de ces projets (scénario 2).

Enfin, deux nouveaux scénarios ont été formulés par la SCTL en alternative à la proposition des fermiers. Le scénario 3 est strictement dicté par une logique "d'entreprise " et cherche à "faire de l'argent avec le bois ". Le scénario 4 vise à concilier les projets individuels avec un projet collectif SCTL, en rentabilisant l'exploitation forestière mais en intervenant chez tous les fermiers qui le souhaitent. Le scénario 3 émanait de gérants davantage soucieux de l'équilibre budgétaire de la SCTL et du GFA Larzac que de la maîtrise de l'enrésinement des parcours. Il est focalisé sur un aménagement forestier contraint par une trésorerie annuelle positive et exclusivement fondé sur la valeur marchande du bois "bord de piste". En revanche, le scénario 4 était porté par des gérants convaincus de la nécessité d'intervenir sur le maximum de surfaces boisées pour agir le plus efficacement possible sur le processus de fermeture du milieu. Il couple à la réalisation des projets des fermiers, des interventions dans les forêts pour lesquelles le fermier n'a pas d'objectif propre et ne s'oppose pas à l'exploitation du bois, en y testant différents niveaux de prélèvement.

Une troisième séance a permis de comparer ces options au travers d'une batterie d'indicateurs élaborés par les participants et calculés par le modèle aux deux niveaux d'organisation mis en confrontation (Simon et Étienne, 2009). L'interface cartographique donne une idée de l'évolution du territoire en terme de fermeture du couvert arboré, de progression des ligneux ou de productivité des parcours. L'interface graphique (figure 3) permet de visualiser l'évolution de multiples indicateurs à l'échelle des exploitations agricoles - fourrage disponible et consommé par type de ressource, besoins du troupeau non satisfaits, surface pâturable, charge de travail comme de la SCTL - volume sur pied en pin et en chêne marchand et non marchand, surfaces aménagées selon chaque type de sylviculture, trésorerie.

\section{Discussion}

\section{Intérêts et limites du modèle}

Le modèle est fondé sur une représentation simplifiée des relations fonctionnelles entre modalités d'utilisation de l'espace 


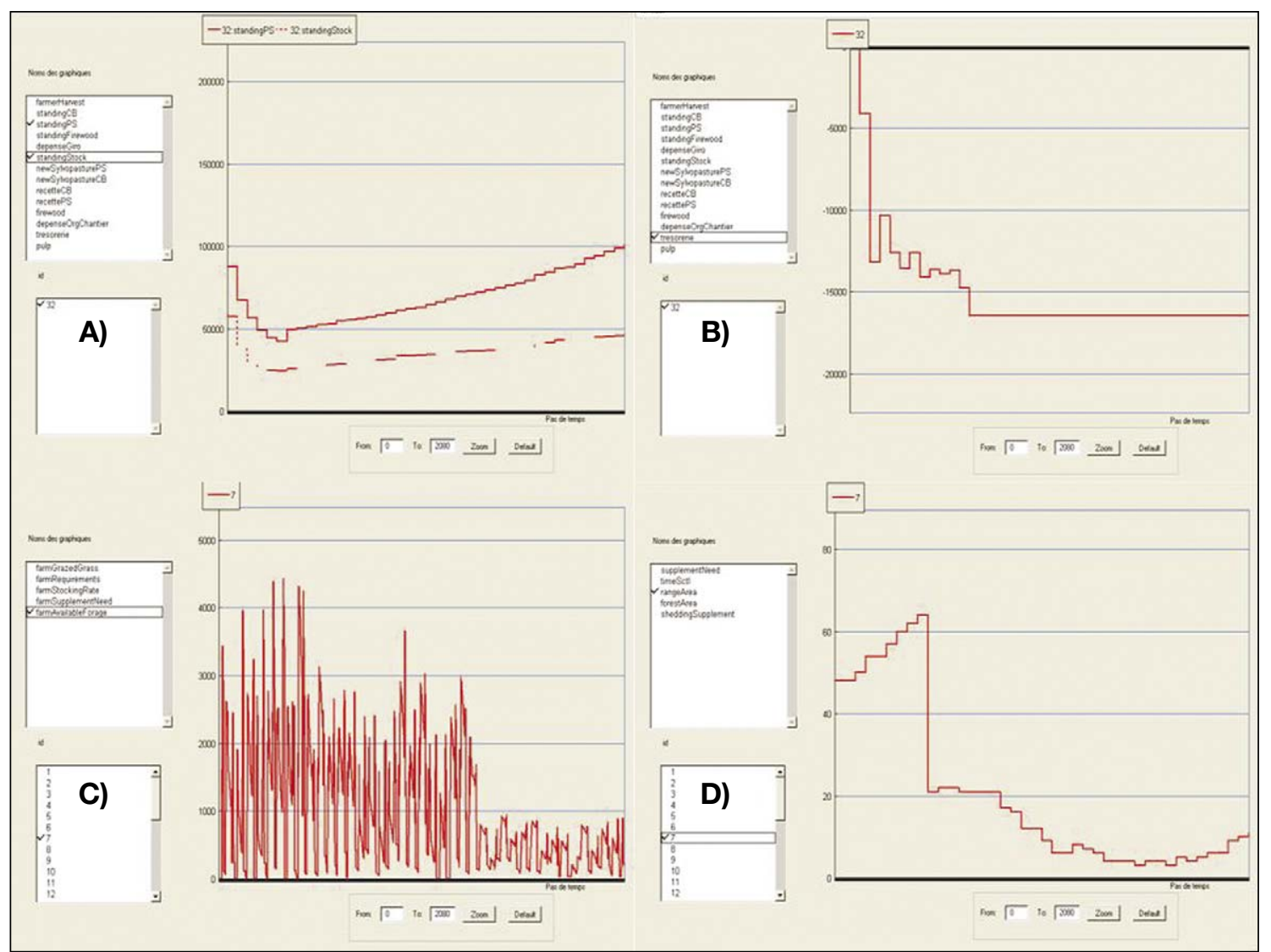

Figure 3. Exemples de sorties graphiques permettant de confronter les résultats d'un scénario à l'échelle du massif forestier.

Figure 3. Chart outputs making it possible to confront the impacts of a scenario at both forest massif scale.

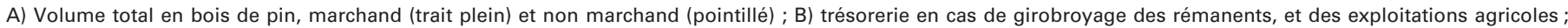
C) surface totale aménagée dans les forêts de pin (trait plein) et de chêne (pointillé); D) surface totale pâturable.

par l'élevage et dynamique agroécologique des couverts végétaux. Il présente d'importantes limites méthodologiques. Les simulations sur les exploitations sont faites à fonctionnement constant: lots d'animaux fixes, règles fixes pour le facteur travail, règles fixes pour l'utilisation de l'espace (à l'exception des itinéraires techniques sylvicoles). De plus le modèle ne tient compte ni de changements du contexte économique - sauf pour le marché du bois - ni de la transmission des exploitations. Cependant, outre la difficulté d'imaginer de nouveaux systèmes de production pour les installations, le système de location sous forme de baux de carrière par la SCTL fait qu'il n'était pas absurde de conserver un système semblable à celui de l'exploitant prenant sa retraite.

La question de la validité du modèle renvoie à la validation par l'usage : le modèle a effectivement été utilisé par la SCTL et ses fermiers pour envisager des gestions alternatives de leur espace boisé. L'objectif était d'apporter aux acteurs locaux une aide à la réflexion, conformément aux principes qui sous-tendent la modélisation d'accompagnement.

Comme il s'agit d'un modèle très ancré sur des spécificités locales, on peut se poser la question de sa généricité. Étant lui-même largement inspiré du modèle Méjan, la transposition à d'autres contextes et problématiques est envisageable, sous réserve que soient disponibles des références pertinentes dans ces autres contextes. La généricité porte donc plutôt sur la démarche elle-même.

L'originalité du modèle SCTL réside dans sa capacité à rendre compte, à l'échelle d'un territoire, des interactions entre des logiques individuelles d'utilisation de l'espace par les exploitations agricoles et la logique d'un collectif confronté à la gestion d'un massif forestier. C'est donc un cas de figure exemplaire en termes de réflexion sur la déprise agricole, par la présence d'un acteur collectif soucieux d'une gestion durable de son territoire forestier.

\section{Contribution aux enjeux de développement durable}

Le modèle prend explicitement en considération l'évolution des couverts végétaux et rend ainsi compte des dynamiques des milieux et des paysages. En revanche, les dimensions sociale et économique y sont plus implicites. Ainsi, les résultats des simulations indiquant la nature et le volume des bois exploitables pourraient se traduire en termes de chiffre d'affaires et d'emplois locaux potentiels; de même le nombre de journées de pâturage possibles sur les parcours pourrait servir d'indicateur pour sécuriser les systèmes fourragers. Le temps disponible pour couvrir les besoins en bois des éleveurs et leurs décisions de procéder eux-mêmes aux interventions sylvicoles pourrait être 
traduit en indicateurs sociaux sur la charge de travail ou sur la capacité d'autosatisfaction des besoins en énergie.

La question posée par la SCTL sur la valorisation de ses espaces boisés a conduit à une réflexion sur la rentabilisation des interventions sylvicoles ou, du moins, sur un équilibre entre produits et coûts, en considérant simultanément les volets pastoral et sylvicole. Mais la plupart des projets individuels des éleveurs du Larzac sur l'équilibre et la pérennité de la combinaison "intervention sur les arbres/ utilisation pastorale des bois " ne comportent pas d'objectifs sur les arbres eux-mêmes, notamment parce que les fermiers ne sont pas propriétaires des espaces sylvopastoraux. Quant aux projets envisagés par la SCTL, ils sont restés dans les cadres classiques des filières existantes, sans véritable réflexion sur : "quels nouveaux produits pour quelles filières?". La recherche d'innovations techniques articulant mises en valeur pastorale et sylvicole pour augmenter la rentabilité des interventions sur les arbres et agrandir l'espace concerné aurait pu constituer une réponse en termes de durabilité, consolider les différentes mises en valeur - volets économique et social - et favoriser et entretenir la diversité des peuplements - volet environnemental. Ainsi, le modèle aurait pu permettre d'explorer de nouvelles modalités de prélèvement d'arbres et d'usage pastoral en jouant sur la préservation de la qualité des reports sur pied de la ressource pastorale, tout en évitant de dégrader les résultats économiques par des interventions de débroussaillement ou d'élimination de certains bois sans valorisation.

\section{Conclusion}

La modélisation d'accompagnement utilisée apparaît comme une démarche intéressante pour mobiliser un collectif d'acteurs autour d'un futur partagé et envisager des actions concertées pour un développement plus durable.
Il semble possible dans un massif forestier de ce type (grande superficie, une propriété collective) de construire et de tester de nouveaux itinéraires technico-économiques, des productions différentes avec des mises en marché correspondant à ces activités sylvopastorales : valoriser une combinaison de produits bois en installant le pâturage des animaux dans la technique sylvicole pour une rentabilité et durabilité communes. Il est même probable que, dans certaines situations, c'est l'activité sylvicole qui pourrait servir à mettre en place l'activité pastorale complémentaire à la réussite d'une gestion durable de ces espaces. Ces tentatives qui n'ont pu être conduites à cette étape du travail ont été reprises dans le programme du Compte d'affectation spéciale développement agricole et rural (Casdar), en mettant l'accent sur les différentes échelles spatiales (parcelle, exploitation, massif ou territoire local) et temporelles, en particulier en retenant un temps commun (une dizaine d'années) : le moyen terme sylvopastoral (Guérin et Picard, 2005 ; Guérin, 2008).

\section{Remerciements}

Ces travaux ont bénéficié du soutien financier de l'Association de coordination technique agricole (Acta) et du Casdar (programmes "Sylvopastoralisme"), du Cemagref (Institut de recherche pour l'ingénierie de l'agriculture et de l'environnement) et de l'Agence nationale de la recherche (projet ANR-05-PADD-003 TRANS [Transformations de l'élevage et dynamiques des espaces]). Ils n'auraient pas pu aboutir sans la volonté, la patience et l'implication des fermiers du Larzac et des gérants de la SCTL.

\section{Références}

Bousquet F, Bakam I, Proton $\mathrm{H}$, Le Page C. CORMAS: Common-Pool Resources and Multiagent Systems. Lect Notes Artif Int 1998 ; 1416 : 826-38.
Collectif ComMod. Modélisation d'accompagnement. In: Amblard F. et Phan D, eds. Modélisation et simulation multi-agents : applications aux sciences de l'homme et de la société. Londres : Hermès sciences, 2006.

De Crisenoy C, Boscheron D. Un office foncier en France: la société civile des terres du Larzac. Paris : Inra, 1986.

Dedieu B, Servière G, Madelrieux S, Dobremez $\mathrm{L}$, Cournut S. Comment appréhender conjointement les changements techniques et les changements du travail en élevage? Cah Agric 2006 ; 15 : 506-13.

Etienne M. Pine trees - invaders or forerunners in Mediterranean-type ecosystems: a controversial point of view. J Mediterr Ecol 2001; 2 : 221-31.

Etienne M, Le Page C, Cohen M. A step-by-step approach to building land management scenarios based on multiple viewpoints on multiagent system simulations. JArtif Societies Soc Simul 2003; 6: epub. http://jasss.soc. surrey.ac.uk/6/2/2.html.

Franc $A$, Sanders L. Modèles et systèmes multi-agents en écologie et en géographie: état de l'art et comparaison avec les approches classiques. In: Ferrand $\mathrm{N}$, ed. Modèles et systèmes multi-agents pour la gestion de l'environnement et des territoires. Antony : Cemagref. 2000

Guérin G. De la forêt pâturée au sylvopastoralisme. Forêt Mediterr 2008; 29 : 491-6.

Guérin G, Bellon S, Gautier D. Valorisation et maîtrise des surfaces pastorales par le pâturage. Fourrages $2001 ; 166$ : 239-56.

Guérin G, Picard O. L'enjeu technique du sylvopastoralisme : des échelles d'espace et de temps communes à l'élevage et à la sylviculture. In : Georgoudis A, Rosati A, Mosconi C, eds. Animal production and natural resources utilisation in the Mediterranean mountain areas. Wageningen (Pays-Bas): Wageningen Academic Publishers, 2005.

Lepart J, Rousset O, Marty P. Les phénomènes d'accrues: analyser, comprendre et prévoir. Ingénieries, 1999 ; NS Boisements : S59-66.

Simon C. Approche multi-agents pour une gestion pastorale et forestière concertée. Application aux espaces boisés de la Société civile des terres du Larzac. Nancy : École nationale du génie rural, des eaux et forêts, 2004.

Simon C, Etienne M. A companion modelling approach applied to forest management planning with the Société Civile des Terres du Larzac. Environ Modell Softw 2009 ; sous presse. 